\title{
Crianças e adolescentes que consomem alimentos ultraprocessados possuem pior perfil lipídico? Uma revisão sistemática
}

\author{
Do children and adolescents who consume ultra-processed foods \\ have a worse lipid profile? A systematic review
}

Jéssica Batista Beserra (https://orcid.org/0000-0001-9420-0166) ${ }^{1}$

Nathanael Ibsen da Silva Soares (https://orcid.org/0000-0003-1042-6307) ${ }^{1}$

Camila Santos Marreiros (https://orcid.org/0000-0001-6225-1698) ${ }^{1}$

Cecília Maria Resende Gonçalves de Carvalho (https://orcid.org/0000-0002-8707-1447) ${ }^{1}$

Maria do Carmo de Carvalho e Martins (https://orcid.org/0000-0002-9107-2485) ${ }^{1}$

Betânia de Jesus e Silva de Almendra Freitas (https://orcid.org/0000-0002-7797-735X) ${ }^{1}$

Marize Melo dos Santos (http://orcid.org/0000-0003-0699-8062) ${ }^{1}$

Karoline de Macêdo Gonçalves Frota (https://orcid.org/0000-0002-9202-5672) ${ }^{2}$

${ }^{1}$ Departamento de Nutrição, Universidade Federal do Piauí (UFPI)

Teresina PI Brasil.

${ }^{2}$ Departamento de Biofísica

e Fisiologia, UFPI. Av.

Universitária, lado ímpar,

Campus Ministro

Petrônio Portela, Bairro

Ininga. 64049-550

Teresina PI Brasil.

karolfrota@ufpi.edu.br

\begin{abstract}
The increase in the input of ultra-processed ingredients in the food of children and adolescents is related to the development of noncommunicable diseases such as dyslipidemia. The scope of this study was to conduct a systematic review of the literature on the relationship of consumption of ultra-processed foods in the lipid profile of children and adolescents. A search in the PubMed, Scopus, Cochrane and LILACS databases was carried out to locate cross-sectional and longitudinal studies, with or without intervention, in apparently healthy children and/or adolescents, who had the intake of ultra-processed food as an exposure variable and the lipid profile as an outcome. After screening, 14 studies were included, of which nine demonstrated that ultra-processed food consumption was related to increased $L D L-c$, total cholesterol, triglycerides and a reduction in $H D L-c$. Three studies found no relationship and two demonstrated that the increased intake of ready-to-eat cereals was related to the decrease in total cholesterol and LDL-c. There was a high consumption of ultra-processed foods and positive relation with blood lipids among children and adolescents, which calls attention to interventions, such as nutritional education, with a view to reducing the intake of these foods.
\end{abstract}

Key words Industrialized foods, Dyslipidemias, Adolescent, Child
Resumo O aumento da participação de alimentos ultraprocessados na alimentação de crianças e adolescentes está relacionado ao desenvolvimento de agravos não transmissiveis, como dislipidemia. Objetivou-se realizar uma revisão sistemática da literatura sobre a relação do consumo de alimentos ultraprocessados e o perfil lipídico de crianças e adolescentes. Realizou-se uma busca nas bases de dados PubMed, Scopus, Cochrane e LILACS por estudos com desenhos transversais e longitudinais, com ou sem intervenção; em crianças elou adolescentes aparentemente saudáveis, que tivessem a ingestão de alimento ultraprocessado como variável de exposição e o perfil lipídico como desfecho. Após triagem, 14 estudos foram incluídos, destes, nove demonstraram que o consumo de ultraprocessados estava relacionado com o aumento do $L D L-c$, colesterol total, triglicerídeos e diminuição do HDL-c. Três estudos não encontraram nenhuma relação e dois demonstraram que a maior ingestão de cereais prontos estava relacionada com a diminuição de colesterol total e LDL-c. Observou-se elevado consumo de alimentos ultraprocessados e relação positiva com lipídios sanguineos em crianças e adolescentes o que chama atenção para a realização de intervenções, como educação nutricional, com vistas a reduzir a ingestão desses alimentos.

Palavras-chave Alimentos industrializados, Dislipidemia, Adolescente, Criança 


\section{Introdução}

O atual estilo de vida da população mundial e o avanço tecnológico da indústria alimentícia influenciou a transição dos hábitos alimentares de uma dieta baseada em alimentos in natura ou minimamente processados para o alto consumo de alimentos processados e ultraprocessados ${ }^{1}$. Esses produtos são constituídos de vários ingredientes e passam por uma série de etapas durante sua fabricação. Geralmente estão prontos para consumo e, portanto, exigem pouca ou nenhuma preparação culinária, o que os torna acessíveis e convenientes. No entanto, esses alimentos são nutricionalmente desbalanceados, pois apresentam alto conteúdo de gorduras saturadas e trans, elevado índice glicêmico, enquanto que possuem baixo teor de fibras, minerais e vitaminas. Além disso, o consumo desses produtos alimentícios tende a limitar a ingestão de alimentos não processados ou minimamente processados ${ }^{2,3}$.

O consumo de produtos alimentícios processados e ultraprocessados aumentou nas últimas décadas tanto em países desenvolvidos quanto nos em desenvolvimento ${ }^{2,4}$. No Brasil, por exemplo, o seu consumo aumentou de $20,3 \%$ para $32,1 \%$ entre 1987 e $2009^{5}$. A mudança no padrão alimentar não ocorreu apenas entre a população adulta, estudos têm mostrado que porção significativa do valor energético consumido por crianças e adolescentes provém de alimentos ultraprocessados $^{6-8}$.

Essas mudanças alimentares têm sido acompanhadas de correspondentes aumentos na prevalência de agravos não transmissíveis relacionadas à alimentação $0^{9-11}$, a exemplo de distúrbios no perfil lipídico, diretamente associada ao desenvolvimento de doenças cardiovasculares ${ }^{12,13}$. No entanto, os estudos que avaliam a associação entre o consumo desses produtos por crianças e adolescentes e dislipidemias ainda são escassos e inconclusivos $^{10,11}$. O objetivo da presente revisão sistemática foi verificar a relação entre o consumo de alimentos ultraprocessados e o perfil lipídico de crianças e adolescentes.

\section{Métodos}

O presente estudo consiste em uma revisão sistemática da literatura. Dois revisores independentes (JBB e NISS) realizaram uma seleção inicial dos artigos localizados na busca eletrônica por meio da leitura dos títulos e resumos no período de 03/06/2018 a 10/06/2018. Os artigos selecionados nessa etapa foram lidos na íntegra e avaliados de acordo com os critérios de elegibilidade. Um terceiro revisor julgou se os artigos deveriam ser mantidos ou excluídos em situações em que os dois revisores discordassem. As recomendações dos Itens de Relatório Preferencial para Revisões Sistemáticas e Meta-Análises (PRISMA) foram seguidas para esta revisão ${ }^{14}$.

\section{Estratégia de Busca}

A busca foi realizada em bancos de dados eletrônicos mundiais (PubMed, Scopus e Cochrane) e em um banco de dados da América Latina e Caribe (LILACS). Termos relacionados ao perfil lipídico e ao consumo de alimentos ultraprocessados foram utilizados. A pesquisa foi composta de combinações dos seguintes termos: ('ultra processed' OR 'ultraprocessed' $O R$ 'ultra-processed' $O R$ 'ready-to-eat' $O R$ 'ready-to-consume' $O R$ 'industrialized' OR 'fast-food' OR 'fast food' OR 'fastfood' OR 'junk food' OR 'prepared food' OR 'candy' OR 'ice cream' OR 'chocolate' OR 'carbonated beverage' OR 'soft drink' OR 'sweetened beverage' OR 'snacks' OR 'sausage' OR 'hot dog' OR 'burger' OR 'dietary patterns' OR 'dietary behaviors' OR 'dietary habits') AND (lipid metabolism or lipoprotein or triglyceride or cholesterol or dyslipidemia).

Todos os artigos incluídos eram estudos originais realizados em seres humanos. Além da busca eletrônica, os revisores também realizaram uma busca manual na lista de referências de cada estudo incluído, para identificar estudos potencialmente relevantes que não haviam sido alcançados na busca inicial. Os detalhes da seleção dos estudos estão dispostos na Figura 1.

\section{Critérios de Elegibilidade}

Para ser elegível, os estudos tinham que cumprir os seguintes critérios: avaliar a exposição (consumo de alimentos ultraprocessados) e o desfecho (perfil lipídico) durante a infância $(0 \mathrm{a}$ 9 anos) ou adolescência ( 10 a 19 anos) e não se tratar de estudos envolvendo grupos portadores de doenças ou condições de saúde específicas. Foram elegíveis estudos transversais e longitudinais, com ou sem intervenção, sem limite para o ano de publicação. A variável de exposição foi a ingestão de qualquer alimento ultraprocessado, conforme definido na classificação da NOVA 3 . 


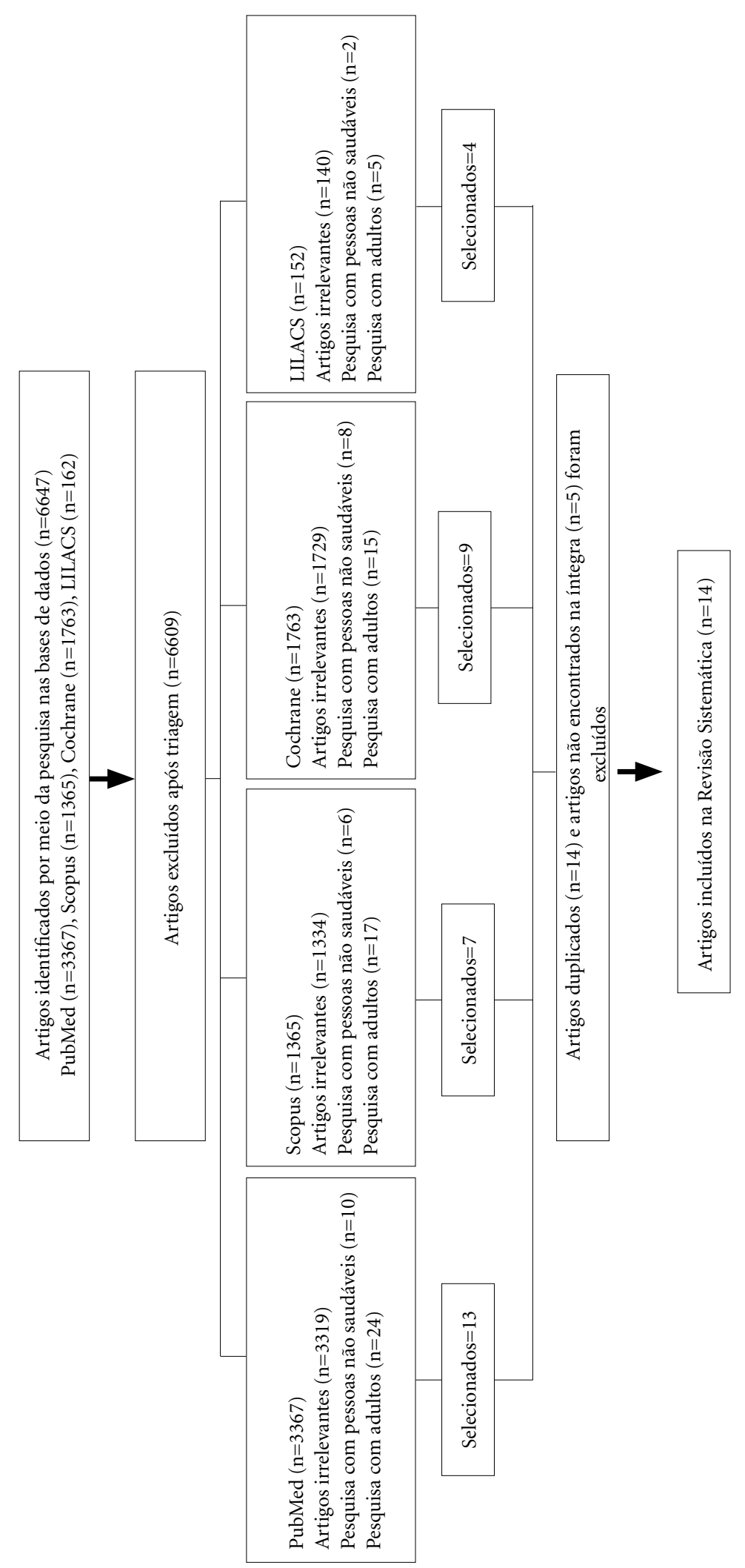

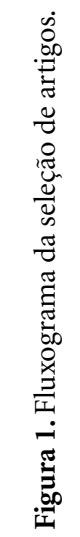




\section{Análise dos dados}

Os artigos selecionados foram analisados e desses foram extraídos os seguintes dados: país e ano de publicação, desenho do estudo, tamanho da amostra, sexo dos participantes, faixa etária avaliada, métodos e instrumentos utilizados para medir as variáveis de exposição e desfecho, variáveis utilizadas para controle de confundimento e principais descobertas.

A qualidade geral e metodológica dos estudos observacionais, incluindo avaliação do viés, foi avaliada de acordo com a Declaração STROBE (Fortalecimento do Relatório de Estudos Observacionais em Epidemiologia) ${ }^{15}$. A declaração CONSORT (Padrões Consolidados de Ensaios de Relatórios $)^{16}$ foi usada para avaliar os estudos de intervenção. A pontuação máxima que pode ser obtida é de 22 pontos na avaliação do STROBE e 25 pontos no CONSORT; dos quais 9 e 10 pontos, respectivamente, estão relacionados à seção de "métodos" dos estudos. Além disso, foi aplicado o teste Mann-Whitney (dados não paramétricos) para comparar os scores da qualidade geral e metodológica dos estudos, usando o software SPSS, versão 20, com o valor de p estabelecido em $5 \%(p<0,05)$.

\section{Resultados}

Foram identificados 6.647 artigos por meio de pesquisa nas seguintes bases de dados: PubMed $(\mathrm{n}=3.367)$, Scopus $(\mathrm{n}=1.365)$, Cochrane $(\mathrm{n}=$ $1.763)$ e LILACS $(\mathrm{n}=152)$. No processo de triagem, os critérios de inclusão foram analisados e os artigos duplicados $(n=14)$ removidos. Ao final, 14 artigos foram considerados elegíveis para esta revisão sistemática. O Quadro 1 mostra as características dos artigos incluídos.

Dentre os 14 artigos selecionados, seis avaliaram a associação entre consumo de bebidas açucaradas e perfil lipídico ${ }^{22-25,27,29}$, cinco exploraram a associação entre grupos de alimentos ultraprocessados e perfil lipídico ${ }^{10,17,18,26,28}$ e três avaliaram a associação entre consumo de alimentos ultraprocessados específicos ("snacks", sobremesas, pizza e cereais prontos para consumo) e concentrações lipídicas ${ }^{19-21}$.

Entre os seis estudos que avaliaram a relação entre o consumo de bebidas açucaradas e gordura corporal, três encontraram associação positiva entre a ingestão deste tipo de bebida e as concentrações de triglicerídeos ${ }^{22,24,27}$, sendo que dois deles também demonstraram associação positiva entre o consumo de bebidas açucaradas e a concentração de colesterol total ${ }^{10,29}$. Além disso, dois estudos mostraram associação negativa entre a ingestão da bebida em questão e os níveis de HDL-c ${ }^{22,25}$. No entanto, em estudo transversal de Eloranta et al..$^{23}$ realizado com 408 crianças com idade entre 6 e 8 anos, não foi verificada associação entre o consumo de bebidas açucaradas e níveis sanguíneos de lipídios.

Entre os cinco trabalhos que avaliaram a associação entre o consumo de grupos de alimentos ultraprocessados e o perfil lipídico, um estudo longitudinal que envolveu seguimento de crianças e adolescente por 3 anos e meio, encontrou associação positiva entre o consumo de fast foods e as concentrações de triglicerídeos naqueles indivíduos que passaram do menor ao maior quartil de consumo desses alimentos ${ }^{28}$. Em outro trabalho longitudinal realizado por Rauber et al. ${ }^{10}$ em crianças com idade inicial entre três e quatro anos, o consumo de produtos ultraprocessados durante a idade pré-escolar foi preditor de aumento do colesterol total e LDL-c na idade escolar.

Por outro lado, os autores McCourt et al. ${ }^{26}$ observaram em seu estudo, que envolveu o seguimento de 487 adolescentes por 10 anos, associação negativa entre o consumo de dieta sweet tooth, caracterizada pelo alto consumo de pudins, chocolates e confeitos, e concentrações de HDL -c, e associação positiva entre o consumo desses alimentos e níveis de LDL-c. Em contrapartida, o estudo transversal de Petridou et al. ${ }^{17}$ e a pesquisa longitudinal de intervenção com educação nutricional envolvendo pais de crianças de sete meses realizada por Rasanen et al. ${ }^{18}$ não demonstraram associação entre o consumo de grupos de alimentos ultraprocessados e concentrações lipídicas sanguíneas.

Entre os três estudos que avaliaram a associação entre consumo de outros alimentos ultraprocessados e perfil lipídico, Van Horn et al. ${ }^{19} \mathrm{em}$ estudo de intervenção com educação nutricional com duração de sete anos envolvendo crianças com idade inicial entre oito e dez anos mostrou associação positiva entre o consumo de snacks, sobremesas e pizzas e as concentrações de LDL -c em garotos. Por outro lado, Albertson et al. ${ }^{20}$ e Gibson et al. ${ }^{21}$ observaram em seus estudos associação positiva entre o consumo de cereais prontos para consumo e melhora do perfil lipídico caracterizada pela diminuição dos níveis de colesterol total e LDL-c.

Em relação à qualidade dos estudos selecionados, os scores médios corresponderam a 17,6 e 


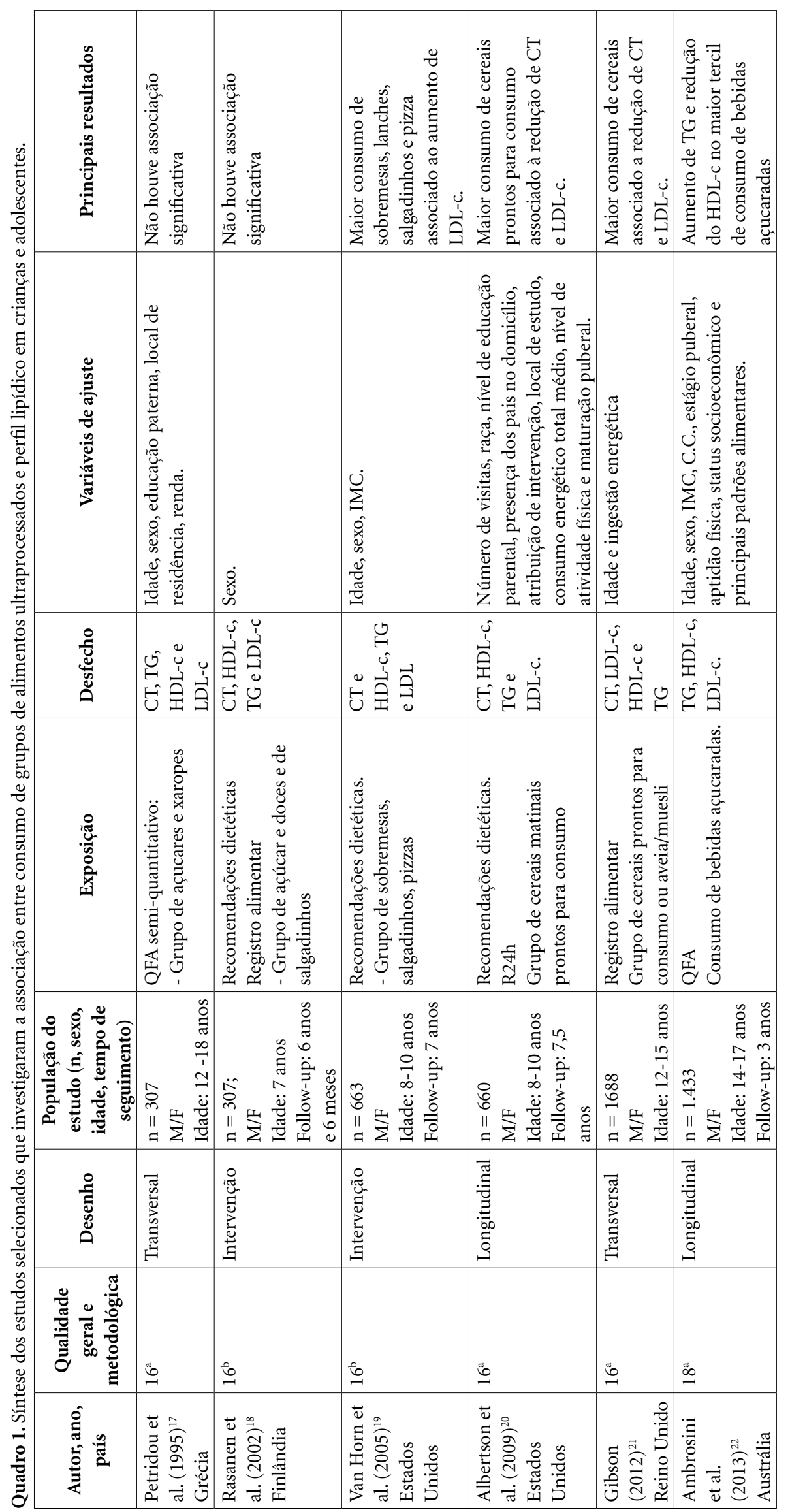




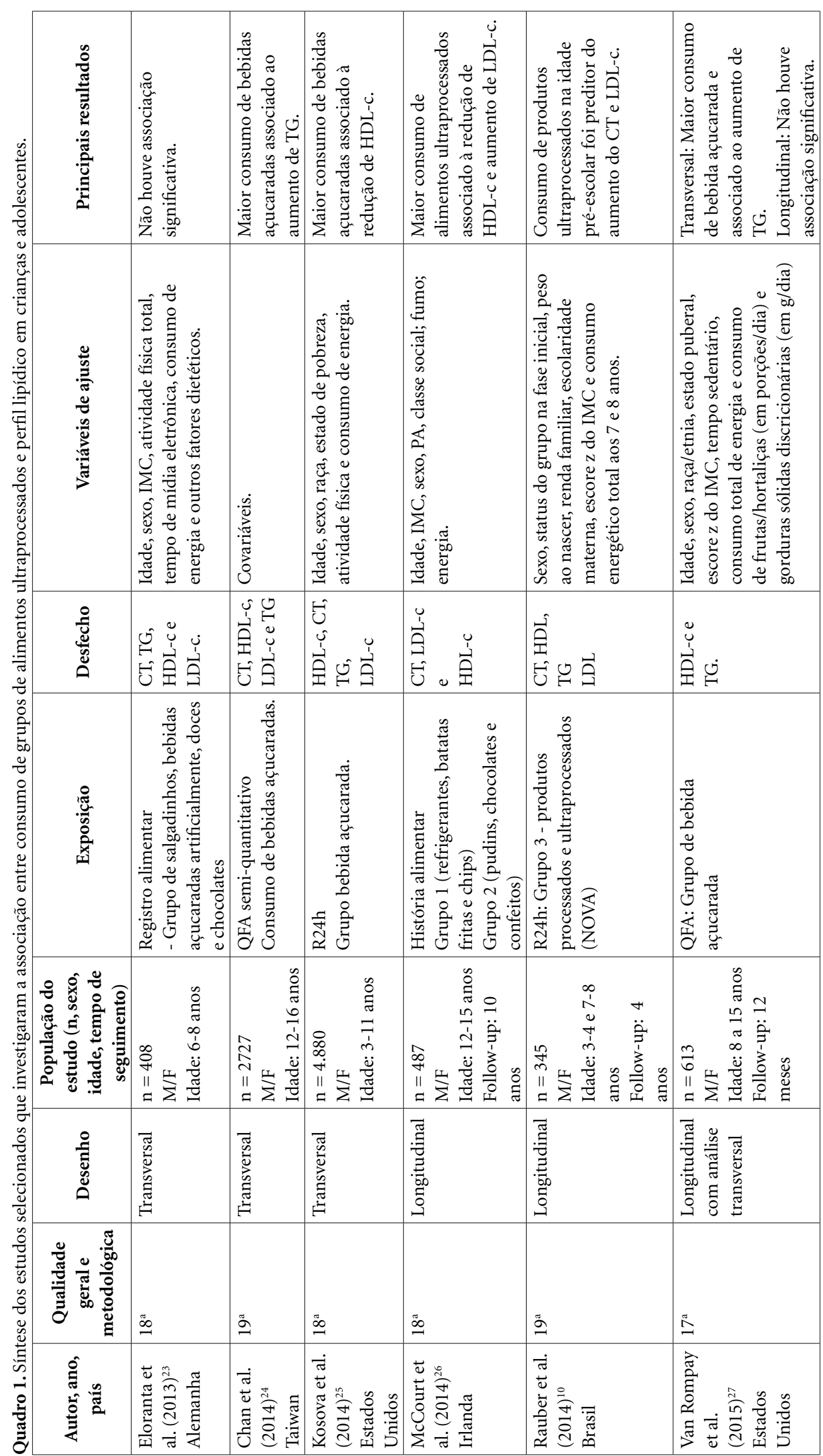




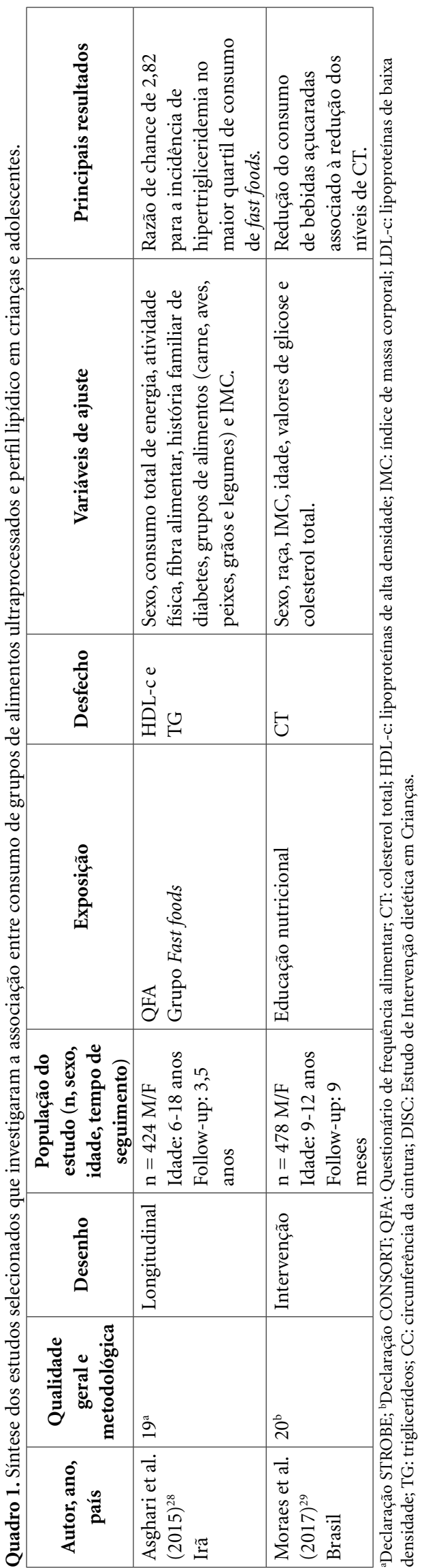

17,3 pontos, respectivamente, para os estudos observacionais $(\mathrm{n}=11)$ e de intervenção $(\mathrm{n}=3)$. A pontuação média alcançada na seção "Métodos" foi de 7 pontos para estudos avaliados usando a Declaração STROBE e a Declaração CONSORT.

\section{Discussão}

$\mathrm{Na}$ presente revisão sistemática a maioria dos trabalhos que investigou o efeito do consumo de alimentos ultraprocessados em crianças e adolescentes, encontrou piora nos parâmetros relacionados ao perfil lipídico. Os hábitos alimentares formados na infância e adolescência geralmente persistem na idade adulta, e podem contribuir para anos de exposição aos malefícios do consumo de alimentos ultraprocessados, o que eleva o risco de dislipidemias, conforme demonstrado nos estudos selecionados ${ }^{30}$.

Em relação aos efeitos do consumo de bebidas açucaradas, a maioria dos trabalhos demonstrou piora nos parâmetros relacionados ao perfil lipídico dos indivíduos avaliados. Estudos têm mostrado que a ingestão de bebidas açucaradas, além de promover desequilíbrio energético, também está ligada a maior risco de dislipidemia por meio do acúmulo de gordura ectópica, adiposidade visceral e hipertrigliceridemia ${ }^{31,32}$.

Tais distúrbios parecem ocorrer devido à elevada quantidade de hidratos de carbono rapidamente absorvíveis presentes nessas bebidas que elevam a lipogênese de novo hepática resultando no aumento dos níveis de triglicerídeos e LDL-c e diminuição das concentrações de HDL-c. Além disso, os carboidratos simples aumentam a glicemia, promovendo elevação da insulinemia que, por sua vez, ativa os fatores de transcrição que induzem a síntese de ácidos graxos e trigliceríde$\mathrm{os}^{33,34}$.

Os mesmos mecanismos podem ser levados em consideração para explicar as associações encontradas nos estudos avaliados que analisaram os efeitos da ingestão de alimentos ultraprocessados do grupo de açúcares e doces, como chocolates e sobremesas em geral, e distúrbios no perfil lipídico das crianças e adolescentes estudados, já que esses alimentos também são acrescidos de açúcares em seu processamento ${ }^{35}$.

Além da elevada quantidade de açúcar, as indústrias alimentícias adicionam grandes quantidades de sódio e gorduras como estratégias de melhoramento da formulação de alimentos ultraprocessados $^{36}$. Esses ingredientes também estão relacionados com distúrbios nos níveis li- 
pídicos no sangue, conforme demonstrado nos estudos encontrados. O sódio, por exemplo, tem sido associado ao aumento das concentrações de cortisol, hormônio que induz a diminuição dos níveis sanguíneos de $\mathrm{HDL}^{37}$.

As gorduras saturadas e trans também estão intimamente relacionadas com as concentrações lipídicas, e estão associadas a um perfil lipídico pró-aterogênico. A gorduras saturadas ocasionam o aumento das concentrações de LDL-c e as gorduras trans, além de induzirem o aumento dessa fração lipídica, causam redução das concentrações de HDL-c $c^{38}$.

Os ácidos graxos trans são formados pela hidrogenação de óleos vegetais, prática bastante comum na produção de produtos alimentícios ultraprocessados. O consumo desse tipo de gordura está relacionado ao aumento de LDL-c e consequente aumento do risco cardiovascular devido à supressão da atividade do receptor dessa fração lipídica, o que resulta na elevação das suas concentrações. As gorduras do tipo trans causam ainda a inibição do funcionamento da paraoxonase, enzima que está associada com a prevenção da oxidação lipídica ${ }^{39,40}$.

Já a redução das concentrações de HDL-c ocorre porque a gordura trans induz o aumento da atividade da proteína de transferência de éster de colesterol, relacionada à etapa fundamental do transporte reverso desse composto. A gordura trans também aumenta o catabolismo do principal tipo de proteína presente nas moléculas de HDL-c, a Apo A1 ${ }^{41,42}$.

Diferente dos outros trabalhos apresentados na presente revisão sistemática, pesquisas realizada por Albertson et al. ${ }^{20} \mathrm{e}$ Gibson et al. ${ }^{21}$ constataram que a maior ingestão de cereais prontos para consumo estava associado à melhora do perfil lipídico dos indivíduos avaliados, com menores níveis de colesterol total e LDL-c. Uma possível explicação para esse resultado é o fato de que, nos estudos em questão, o termo "cereal pronto para consumo" foi utilizado para todos os tipos de cereais frios, tanto não adoçados quanto adoçados, e tanto refinados quanto integrais. Supõese então, que dentre os tipos de cerais estudados, a maioria, mesmo sendo prontos para consumo, possuía boa qualidade nutricional.

Essa evidência é indicada pelos demais resultados do trabalho que demonstraram que a maior frequência de ingestão de cereais prontos para consumo estava associada ao maior consumo de fibras, vitaminas C e D, folato, cálcio, ferro e zinco, nutrientes que estão associados com a melhora do perfil lipídico, além de estar relacionada com redução da ingestão de colesterol, e da porcentagem de energia proveniente de gorduras ${ }^{43,44}$.

Os estudos de Petridou et al. ${ }^{17}$, Rasanen et al. ${ }^{18}$ e Eloranta et al.$^{23}$, não encontraram associação entre o consumo de produtos ultraprocessados e perfil lipídico, sendo que algumas limitações apontadas abaixo, podem ter comprometido os resultados encontrados.

Petridou et al. ${ }^{17}$, em estudo transversal realizado na Grécia com 307 adolescentes, não encontraram associação entre o consumo de alimentos do grupo de açúcares e xaropes e as concentrações de colesterol total, triglicerídeos, HDL-c e LDL-c. Os autores relatam a dificuldade inerente de demonstrar associações entre o consumo dietético e os lipídios séricos, principalmente porque os componentes endógenos, como os relacionados ao metabolismo podem sobrepor os componentes exógenos como a dieta, dependendo da quantidade do componente ingerido.

No trabalho de Rasanen et al. ${ }^{18}$ que objetivou avaliar os padrões alimentares e as concentrações lipídicas de crianças após 6 anos e 5 meses de intervenção realizada com educação nutricional para os pais, quando comparadas ao grupo cujos pais não receberam intervenção, não observou-se associação entre o consumo de alimentos do grupo de açúcares e doces e as concentrações sanguíneas de colesterol total, triglicerídeos, HDL-c e LDL-c. Algumas limitações podem explicar os achados da pesquisa. Primeiramente, os autores não avaliaram os grupos intervenção e controle separadamente em relação aos parâmetros envolvidos no trabalho, realizando a divisão por tipo de "padrão alimentar" segundo a análise de clusters. Além disso, os pesquisadores relataram que o recordatório alimentar era realizado logo após a intervenção, o que pode ter alterado a fidedignidade dos dados.

No estudo de Eloranta et al..$^{23}$, realizado na Finlândia com 408 crianças, o consumo de salgadinhos, bebidas açucaradas artificialmente, doces e chocolates não estavam associados às concentrações de colesterol total, triglicerídeos, HDL-c e LDL-c. O estudo aponta que o registro alimentar e o nível de atividade física foram relatados pelos pais, o que pode ter causado erros de declaração. Além disso, as ocasiões de alimentação (café da manhã, almoço, jantar e lanches) foram definidas com base nas impressões da nutricionista clínica e dos pais.

Vale ressaltar que os escores referentes à qualidade dos estudos incluídos na presente revisão, avaliados por meio das Declarações STROBE e CONSORT, foram considerados bons, inclusive 
os referentes aos estudos que não encontraram associação entre os parâmetros estudados (Petridou et al. ${ }^{17}=16$; Rasanen et al. ${ }^{18}=16$; Eloranta et al. $^{23}=18$ ). Além disso, não houve diferença significativa nos escores médios de qualidade entre os estudos que encontraram ou não uma associação ( $\mathrm{p}>0,05)$.

Algumas limitações dificultaram a avaliação e discussão dos dados da presente revisão sistemática. Dentre elas destaca-se a utilização de diferentes tipos de métodos utilizados para investigar o consumo de alimentos, que podem ter resultado em subestimação ou superestimação da ingestão alimentar dos indivíduos estudados. Além disso, a diferença entre os pontos de corte utilizados para avaliar o perfil lipídico dos indivíduos podem ter influenciado a análise dos resultados encontrados pelos autores.

A divergência entre os desenhos dos estudos, já que a revisão envolveu pesquisas transversais e longitudinais, de intervenção ou não, também limitaram a análise e discussão dos dados. Vale ressaltar ainda, que alguns estudos não ajustaram os resultados levando em consideração alterações características da puberdade, o que pode ter comprometido os resultados encontrados, tendo em vista que nessa etapa da vida alterações metabólicas e fisiológicas alteram parâmetros como sensibilidade à insulina e níveis lipídicos ${ }^{45-47}$. Alguns estudos apontam que essas alterações podem ocorrer por influência das mudanças hormonais durante a maturação sexual, em especial na concentração plasmática de testosterona e estradiol $^{48,49}$.

Dessa forma, os resultados dos estudos avaliados nesta revisão sistemática fornecem evidências do efeito prejudicial do consumo de alimentos ultraprocessados sobre o perfil lipídico de crianças e adolescentes. No entanto, mais estudos nessa temática e faixa etária são necessários, principalmente aqueles com delineamento longitudinal que podem elucidar a associação entre os dois parâmetros de forma mais eficaz. Existe ainda uma lacuna na avaliação dietética por não considerar a extensão e o nível de processamento dos alimentos, que tem sido relacionado aos diversos fatores de risco para doenças crônicas não transmissíveis, incluindo as dislipidemias. Além disso, ressalta-se a importância de uma classificação padronizada internacionalmente que considere o nível de processamento do alimento para promover uma melhor comparabilidade dos estudos.

\section{Colaboradores}

JB Beserra e NIS Soares participaram da concepção e projeto, análise e interpretação dos dados e redação do artigo. CS Marreiros participou da análise e interpretação dos dados e redação do artigo. CMRG Carvalho e MCC Martins participaram da concepção e projeto e revisão do artigo. BJSA Freitas e MM Santos participaram da revisão do artigo. KMG Frota participou da concepção e projeto, revisão do artigo e aprovação final da versão a ser publicada. 


\section{Referências}

1. Moubarac JC, Martins APB, Claro RM, Levy RB, Cannon G, Monteiro CA. Consumption of ultra -processed foods and likely impact on human health. Evidence from Canada. Public Health Nutr 2013; 16(12):2240-2248.

2. Louzada ML, Martins AP, Canella D, Baraldi L, Levy R, Claro R, Moubarac JC, Cannon G, Monteiro CA. Ultra-processed foods and the nutritional dietary profile in Brazil. Rev Saude Publica 2015; 49:38.

3. Monteiro CA, Cannon G, Levy R, Moubarac JC, Jaime P, Martins AP, Canella D, Louzada M, Parra D. O sistema alimentar: classificação dos alimentos: saúde pública: nova: a estrela brilha. World Nutr 2016; 7(13):28-40.

4. Baraldi LG, Martinez Steele E, Canella D, Monteiro CA. Consumption of ultra-processed foods and associated sociodemographic factors in the USA between 2007 and 2012: evidence from a nationally representative cross-sectional study. BMJ Open 2018; 8(3): $\mathrm{e} 020574$.

5. Martins APB, Levy RB, Claro RM, Moubarac JC, Monteiro CA. Increased contribution of ultra-processed food products in the Brazilian diet (1987-2009). Rev Saúde Pública 2013; 47(4):656-665.

6. Brasil. Ministério da Saúde (MS). Instituto Brasileiro de Geografia e Estatística (IBGE). Ministério do Planejamento, Orçamento e Gestão. Pesquisa de orçamentos familiares 2008-2009: avaliação nutricional da disponibilidade de alimentos no Brasil. Rio de Janeiro: IBGE; 2010.

7. D'Avila HF, Kirsten VR. Energy intake from ultra -processed foods among adolescents. Rev Paul Pediatr 2017; 35(1):54-60.

8. Sparrenberger K, Friedrich RR, Schiffner MD, Schuch I, Wagner MB. Ultra-processed food consumption in children from a Basic Health Unit. J Pediatr (Rio J) 2015; 91(6):535-542.

9. Bigornia SJ, LaValley MP, Noel SE, Moore LL, Ness AR, Newby PK. Sugar-sweetened beverage consumption and central and total adiposity in older children: a prospective study accounting for dietary reporting errors. Public Health Nutr 2015; 18(7):1155-1163.

10. Rauber F, Campagnolo PD, Hoffman DJ, Vitolo MR. Consumption of ultra-processed food products and its effects on children's lipid profiles: a longitudinal study. Nutr Metab Cardiovasc Dis 2015; 25(1):116122.

11. Tavares LF, Fonseca SC, Garcia Rosa ML, Yokoo EM. Relationship between ultra-processed foods and metabolic syndrome in adolescents from a Brazilian Family Doctor Program. Public Health Nutr 2012; 15(1):82-87.

12. Rocha NP, Milagres LC, Longo GZ, Ribeiro AQ, Novaes JF. Association between dietary pattern and cardiometabolic risk in children and adolescents: a systematic review. J Pediatr (Rio J) 2017; 93(3):214-222.

13. Xavier HT, Izar MC, Faria Neto JR, Assad MH, Rocha VZ, Sposito AC, Fonseca FA, Santos JE, Santos RD, Bertolami MC, Faludi AA, Martinez TLR, Diament J, Guimarães A, Forti NA, Moriguchi E, Chagas ACP, Coelho OR, Ramires JAF. V diretriz brasileira de dislipidemias e prevenção da aterosclerose. Arq Bras Cardiol 2013; 101(4 Supl. 1):1-20.
14. Moher D, Liberati A, Tetzlaff J, Altman DG, PRISMA Group. Preferred reporting items for systematic reviews and meta-analyses: the PRISMA statement. PLoS Med 2009; 6(7):e1000097.

15. Vandenbroucke JP, Von Elm E, Altman DG, Gøtzsche PC, Mulrow CD, Pocock SJ, Poole C, Schlesselman JJ, Egger M, STROBE Initiative. Strengthening the Reporting of Observational Studies in Epidemiology (STROBE): explanation and elaboration. Epidemiology 2007; 18(6):805-835.

16. Moher D, Hopewell S, Schulz KF, Montori V, Gøtzsche PC, Devereaux PJ, Elbourne D, Egger M, Altman DG. CONSORT 2010 explanation and elaboration: updated guidelines for reporting parallel group randomised trials. J Clin Epidemiol 2010; 63(8):e1-e37.

17. Pedritou E, Malamou H, Doxiadis S, Pantelakis S, Kanellopoulou G, Toupadaki N, Trichopoulou A, Flytzani V, Trichopoulos D. Blood lipids in Greek adolescents and their relation to diet, obesity, and socioeconomic factors. Ann Epidemiol 1995; 5(4):286-291.

18. Räsänen $M$, Lehtinen JC, Niinikoski $H$, Keskinen S, Ruottinen S, Salminen M, Rönnemaa T, Viikari J, Simell O. Dietary patterns and nutrient intakes of 7-year-old children taking part in an atherosclerosis prevention project in Finland. J Am Diet Assoc 2002; 102(4):518-524.

19. Van Horn L, Obarzanek V, Friedman LA, Gernhofer N, Barton B. Children's adaptations to a fat-reduced diet: the dietary intervention study in children (DISC). Pediatrics 2005; 115(6):1723-1733.

20. Albertson AM, Affenito SG, Bauserman R, Holschuh NM, Eldridge AL, Barton BA. The relationship of ready-to-eat cereal consumption to nutrient intake, blood lipids, and body mass index of children as they age through adolescence. J Am Diet Assoc 2009; 109(9):1557-1565.

21. Gibson S. Micronutrient intakes, micronutrient status and lipid profiles among young people consuming different amounts of breakfast cereals: further analysis of data from the National Diet and Nutrition Survey of Young People aged 4 to 18 years. Public Health Nutr 2003; 6(8):815-820.

22. Ambrosini GL, Oddy WH, Huang RC, Mori TA, Beilin LJ, Jebb SA. Prospective associations between sugar-sweetened beverage intakes and cardiometabolic risk factors in adolescents. Am J Clin Nutr 2013; 98(2):327-334.

23. Eloranta AM, Lindi V, Schwab U, Kiiskinen S, Venalainen T, Lakka HM, Laaksonen DE, Lakka TA. Dietary factors associated with metabolic risk score in Finnish children aged 6-8 years: the PANIC study. Eur J Nutr 2014; 53(6):1431-1439.

24. Chan TF, Lin WT, Huang HL, Lee CY, Wu PW, Chiu YW, Huang CC, Tsai S, Lin CL, Lee CH. Consumption of sugar-sweetened beverages is associated with components of the metabolic syndrome in adolescents. Nutrients 2014; 6(5):2088-2103.

25. Kosova EC, Auinger P, Bremer AA. The relationships between sugar-sweetened beverage intake and cardiometabolic markers in young children. J Acad Nutr Diet 2013; 113(2):219-227. 
26. McCourt HJ, Draffin CR, Woodside JV, Cardwell CR, Young IS, Hunter SJ, Murray LJ, Boreham CA, Gallagher AM, Neville CE, McKinley MC. Dietary patterns and cardiovascular risk factors in adolescents and young adults: the Northern Ireland Young Hearts Project. Br J Nutr 2014; 112(10):1685-1698.

27. Van Rompay MI, McKeown NM, Goodman E, Eliasziw M, Chomitz VR, Gordon CM, Economos CD, Sacheck JM. Sugar-Sweetened Beverage Intake Is Positively Associated with Baseline Triglyceride Concentrations, and Changes in Intake Are Inversely Associated with Changes in HDL Cholesterol over 12 Months in a Multi Ethnic Sample of Children. J Nutr 2015; 145(10):2389-2395.

28. Asghari G, Yuzbashian E, Mirmiran P, Mahmoodi B, Azizi F. Fast food intake increases the incidence of metabolic syndrome in children and adolescents: tehran lipid and glucose study. PLoS One 2015; 10(10):e0139641.

29. Moraes MM, Mediano MFF, Souza RAG, Moura AS, Veiga GV, Sichieri R. Discouraging soft drink consumption reduces blood glucose and cholesterol of Brazilian elementary students: Secondary analysis of a randomized controlled trial. Prev Med 2017; 100:223228.

30. Kaikkonen JE, Mikkilä V, Magnussen CG, Juonala M, Viikari JS, Raitakari OT. Does childhood nutrition influence adult cardiovascular disease risk? - Insights from the Young Finns Study. Ann Med 2013; 45(2):120-128.

31. Richelsen B. Sugar-sweetened beverages and cardiometabolic disease risks. Curr Opin Clin Nutr Metab Care 2013;16(4):478-484.

32. Te Morenga LA, Howatson AJ, Jones RM, Mann J. Dietary sugars and cardiometabolic risk: systematic review and meta-analyses of randomized controlled trials of the effects on blood pressure and lipids $A m J$ Clin Nutr 2014; 100:65-79.

33. Castro C, Corraze G, Pérez-Jiménez A, Larroquet L, Cluzeaud M, Panserat S, Oliva-Teles A. Dietary carbohydrate and lipid source affect cholesterol metabolism of European sea bass (Dicentrarchus labrax) juveniles. Br J Nutr 2015; 114(8):1143-1156.

34. Cassani RSL. What healthy diet is for the prevention of cardiovascular disease: The role nutrients on lipids, overweight, arterial hypertension and subclinical inflammation. Rev Soc Cardiol 2012; 22(2):9-13.

35. Sonestedt E, Hellstrand S, Schulz CA, Wallström P, Drake I, Ericson U, Gullberg B, Hedblad B, OrhoMelander M. The association between carbohydrate -rich foods and risk of cardiovascular disease is not modified by genetic susceptibility to dyslipidemia as determined by 80 validated variants. PLoS One 2015; 10(4): 0126104

36. Tseng M, Neill DB, Teaford SF, Nazmi A. Alternative MyPlate Menus: Effects of Ultra-Processed Foods on Saturated Fat, Sugar, and Sodium Content. J Nutr Educ Behav 2018; 50(3):258-266.

37. Baudrand R, Campino C, Carvajal CA, Olivieri O, Guidi G, Faccini G, Vöhringer PA, Cerda J, Owen G, Kalergis AM, Fardella CE. High sodium intake is associated with increased glucocorticoid production, insulin resistance and metabolic syndrome. Clin. Endocrinol 2014; 80(5):677-684.
38. Santos RD, Gagliardi AC, Xavier HT, Magnoni CD Cassani R, Lottenberg AM. I Diretriz sobre o consumo de gorduras e saúde cardiovascular. Arq Bras Cardiol 2013; 100(1 Supl. 3):1-40.

39. Gayet-Boyer C, Tenenhaus-Aziza F, Prunet C, Marmonier $\mathrm{C}$, Malpuech-Brugère $\mathrm{C}$, Lamarche $\mathrm{B}$, Chardigny JM. Is there a linear relationship between the dose of ruminant trans-fatty acids and cardiovascular risk markers in healthy subjects: results from a systematic review and meta-regression of randomised clinical trials. Br J Nutr 2014; 112(12):1914-1922.

40. Gebauer SK, Chardigny JM, Jakobsen MU, Lamarche B, Lock AL, Proctor SD, Baer DJ. Effects of ruminant trans fatty acids on cardiovascular disease and cancer: a comprehensive review of epidemiological, clinical, and mechanistic studies. Adv Nutr 2011;2(4):332-354

41. Gazzola J, Depin MH. Associação entre consumo de gordura trans e o desenvolvimento de doenças cardiovasculares (DCV). Extensio 2015; 12(20):90-102.

42. Ooi EM, Watts GF, Ng TW, Barrett PHR. Effect of Dietary Fatty Acids on Human Lipoprotein Metabolism: A Comprehensive Update. Nutrients 2015; 7(6):4416-4425.

43. Ranasinghe $\mathrm{P}$, Wathurapatha WS, Ishara $\mathrm{MH}$, Jayawardana R, Galappatthy P, Katulanda P, Constatine GR. Effects of zinc supplementation on serum lipids: a systematic review and meta-analysis. Nutr Metab 2015; 12:26.

44. Zittermann A, Gummert JF, Börgermann J. The role of vitamin D in dyslipidemia and cardiovascular disease. Curr Pharm Des 2011; 17(9):933-42.

45. Eissa MA, Mihalopoulos NL, Holubkov R, Dai S, Labarthe DR. Changes in fasting lipids during puberty. $J$ Pediatr 2016; 170:199-205.

46. Katon JG, Flores YN, Salmeron J. Sexual maturation and metabolic profile among adolescents and children of the Health Worker Cohort Study in Mexico. Salud Publica Mex 2009; 51:219-226.

47. Mascarenhas LP, Leite N, Titski AC, Brito LM, Boguszewski MC. Variability of lipid and lipoprotein concentrations during puberty in Brazilian boys. J Pediatr Endocrinol Metab 2015; 28(1-2):125-131.

48. Laskarzewski PM, Morrison JA, Gutai J, Khoury PR, Glueck CJ. Longitudinal relationships among endogenous testosterone, estradiol, and Quetelet index with high and low density lipoprotein cholesterols in adolescent boys. Pediatr Res 1983; 17:689-698

49. Morrison JA, Barton BA, Biro FM, Sprecher DL. Sex hormones and the changes in adolescent male lipids: longitudinal studies in a biracial cohort. $J$ Pediatr 2003; 142:637-642.

Artigo apresentado em 18/07/2018

Aprovado em 15/04/2019

Versão final apresentada em 17/04/2019 
